\title{
Reichenbachian Common Cause Systems
}

\author{
G. Hofer-Szabó \\ Department of Philosophy \\ Technical University of Budapest \\ e-mail: gszabo@hps.elte.hu \\ Miklós Rédei \\ Department of History and Philosophy of Science \\ Eötvös University, Budapest \\ e-mail: redei@ludens.elte.hu \\ Forthcoming in \\ International Journal of Theoretical Physics
}

\begin{abstract}
A partition $\left\{C_{i}\right\}_{i \in I}$ of a Boolean algebra $\mathcal{S}$ in a probability measure space $(\mathcal{S}, p)$ is called a Reichenbachian common cause system for the correlated pair $A, B$ of events in $\mathcal{S}$ if any two elements in the partition behave like a Reichenbachian common cause and its complement, the cardinality of the index set $I$ is called the size of the common cause system. It is shown that given any correlation in $(\mathcal{S}, p)$, and given any finite size $n>2$, the probability space $(\mathcal{S}, p)$ can be embedded into a larger probability space in such a manner that the larger space contains a Reichenbachian common cause system of size $n$ for the correlation. It also is shown that every totally ordered subset in the partially ordered set of all partitions of $\mathcal{S}$ contains only one Reichenbachian common cause system. Some open problems concerning Reichenbachian common cause systems are formulated.
\end{abstract}

\section{Reichenbach's notion of common cause}

Let $(\mathcal{S}, p)$ be a classical probability space with Boolean algebra $\mathcal{S}$ of random events and probability measure $p$ on $\mathcal{S}$. If the joint probability $p(A \cap B)$ of $A$ and $B$ is greater than the product of the single probabilities, i.e. if

$$
p(A \cap B)>p(A) p(B)
$$

then the events $A$ and $B$ are said to be (positively) correlated and the quantity

$$
\operatorname{Corr}(A, B) \equiv p(A \cap B)-p(A) p(B)
$$

is called the correlation of $A$ and $B$.

According to Reichenbach [13], Section 19, a probabilistic common cause of a correlation such as (1) is an event $C$ (common cause) that satisfies the four conditions specified in the next definition.

Definition $1 C$ is a Reichenbachian common cause of the correlation (1) if the following (independent) conditions hold:

$$
\begin{aligned}
p(A \cap B \mid C) & =p(A \mid C) p(B \mid C) \\
p\left(A \cap B \mid C^{\perp}\right) & =p\left(A \mid C^{\perp}\right) p\left(B \mid C^{\perp}\right) \\
p(A \mid C) & >p\left(A \mid C^{\perp}\right) \\
p(B \mid C) & >p\left(B \mid C^{\perp}\right)
\end{aligned}
$$

where $p(X \mid Y)=p(X \cap Y) / p(Y)$ denotes the conditional probability of $X$ on condition $Y, C^{\perp}$ denotes the complement of $C$ and it is assumed that none of the probabilities $p(X),\left(X=A, B, C, C^{\perp}\right)$ is equal to zero. 
We shall occasionally refer to conditions (3)-(6) as "Reichenbach(ian) conditions". It is standard terminology to call (3)-(4) "screening-off" conditions and to say that $C$ (and also $C^{\perp}$ ) screens off the correlation between $A$ and $B$. To exclude trivial common causes we call a common cause $C$ proper if it differs from both $A$ and $B$ by more than a measure zero event. In what follows "common cause" will always mean a proper common cause.

Reichenbach prooves the following proposition.

Proposition 1 If the events $A, B, C$ satisfy the Reichenbachian conditions (3)-(6) then there is a positive correlation between $A$ and $B$.

The significance of Proposition 1 is that it shows in what sense a common cause explains a correlation: from the assumption that $A, B$ and $C$ satisfy the Reichenbachian conditins one can derive (equivalently: predict) that $A$ and $B$ are (positively) correlated - this is an instance of explanation in the sense of Hempel.

Reichenbach's proof of Proposition 1 is based on the following Lemma, which, for later purposes, we spell out in a slightly more general form than used by Reichenbach. Before stating the lemma let us recall that the set of events $\left\{C_{i} \in \mathcal{S} \mid i \in I\right\}$ is a partition of $\mathcal{S}$ if $\cup_{i} C_{i}=\Omega$ ( $\Omega$ being the unit in $\mathcal{S}$ ) and $C_{i} \cap C_{j}=\emptyset$ if $i \neq j$.)

Lemma: Let $\left\{C_{i}\right\}_{i \in I}$ be a partition of $\mathcal{S}$ and let $A, B \in \mathcal{S}$ be arbitrary elements. If $p\left(A \cap B \mid C_{i}\right)=$ $p\left(A \mid C_{i}\right) p\left(B \mid C_{i}\right)$ for all $i \in I$ then we have

$$
p(A \cap B)-p(A) p(B)=\frac{1}{2} \sum_{i \neq j} p\left(C_{i}\right) p\left(C_{j}\right)\left[p\left(A \mid C_{i}\right)-p\left(A \mid C_{j}\right)\right]\left[p\left(B \mid C_{i}\right)-p\left(B \mid C_{j}\right)\right]
$$

Applying Lemma (proof of which is left to the reader) with $C_{1}=C$ and $C_{2}=C^{\perp}$ one obtains

$$
p(A \cap B)-p(A) p(B)=p(C) p\left(C^{\perp}\right)\left[p(A \mid C)-p\left(A \mid C^{\perp}\right)\right]\left[p(B \mid C)-p\left(B \mid C^{\perp}\right)\right]
$$

which is the formula Reichenbach uses in showing Proposition 1.

Eq. (8) implies that $\operatorname{Corr}(A, B)$ is indeed positive if (5)-(6) hold. Eq. (8) also shows, however, that for $\operatorname{Corr}(A, B)$ to be positive (5)-(6) are sufficient but not necessary: positivity of $\operatorname{Corr}(A, B)$ is implied by the positivity of the right hand side of $(8)$; hence, what is decisive from the point of view of the explanatory power of the comon cause is that $\left[p(A \mid C)-p\left(A \mid C^{\perp}\right)\right]$ and $\left[p(B \mid C)-p\left(B \mid C^{\perp}\right)\right]$ have the same sign. It also is clear from (8) that a common cause can explain negative correlations in just the same way as it can explain positive ones: if $C$ and $C^{\perp}$ are such that screening off conditions (3)-(4) hold and $\left[p(A \mid C)-p\left(A \mid C^{\perp}\right)\right]$ and $\left[p(B \mid C)-p\left(B \mid C^{\perp}\right)\right]$ have opposite signs, then the right hand side of (8) is negative, hence existence of such a $C$ entails the negative correlation. All what follows can be modified trivially in order to cover the case of negative correlation, and all statements presented below remain valid in the case of negative correlations; however, to simplify notation we restrict ourselves to positive correlations.

To sum up: the intuitive idea behind explaining by a Reichenbachian common cause a correlation between $A$ and $B$ in a statistical ensemble is that one should be able to cut the statistical ensemble by a pair of orthogonal events $\left(C\right.$ and $\left.C^{\perp}\right)$ into two disjoint parts in such a way that (i) the correlation disappears in both of the resulting subensembles (this is expressed by the two screening off conditions); and (ii) one of the subensembles should increase the probability of both $A$ and $B$ (which is the content of the requirement of $\left[p(A \mid C)-p\left(A \mid C^{\perp}\right)\right]$ and $\left[p(B \mid C)-p\left(B \mid C^{\perp}\right)\right]$ having the same sign).

\section{The notion of a Reichenbachian common cause system}

It is easy to see that there exist common cause incomplete probability spaces $(\mathcal{S}, p)$, i.e. probability spaces that contain a pair of correlated events without containing a (proper) common cause of the correlation. Existence of such common cause incomplete probability spaces can be a threat to what has become called Reichenbach's Common Cause Principle (RCCP): Given a correlation $\operatorname{Corr}(A, B)>$ 0 , either there is a direct causal influence between $A$ and $B$ that can be held responsible for the correlation, or there exists a common cause in the sense of Definition 1 that explains the correlation. So if one sees a correlation between $A$ and $B$ and has good reasons to think that the correlated events $A, B$ in $(\mathcal{S}, p)$ cannot influence each other causally and yet there exists no common cause in $\mathcal{S}$ of the correlation $\operatorname{Corr}(A, B)$, then the suspicion arises that RCCP might not hold.

Confronted with a common cause incomplete probability space $(\mathcal{S}, p)$ in which a direct causal influence between the correlated events is excluded, one can have in principle two strategies aiming at saving RCCP: One may try to argue that $\mathcal{S}$ is not "rich enough" to contain a common cause 
but there might exist a larger $\left(\mathcal{S}^{\prime}, p^{\prime}\right)$ that already contains a common cause of the correlation (see Definition 3 below for what it means to enlarge $(\mathcal{S}, p)$ into $\left.\left(\mathcal{S}^{\prime}, p^{\prime}\right)\right)$. It was shown in a previous paper that this strategy always works in the sense that it is always possible to enlarge $(\mathcal{S}, p)$ in such a way that the enlarged space already contains an event $C$ that satisfies the Reichenbachian conditions (see $[4])$.

Another natural idea is to suspect that the correlation between $A$ and $B$ is not due to a single factor but may be the cumulative result of a (possibly large) number of different "partial common causes", none of which can in and by itself yield a complete common-cause-type explanation of the correlation, all of which, taken together, can however account for the entire correlation. Explaining a correlation by such a system of partial common causes would mean that one can partition the statistical ensemble into more than two subensembles in such a manner that (i) the correlation disappears in each of the subensembles, (ii) any pair of such subensembles behaves like the two subensembles determined by the pair $C, C^{\perp}$ in the Definition 1 of common cause and (iii) the totality of "partial common causes" explains the correlation in the sense of entailing it. A mathematically explicit formulation of this idea is spelled out in the next definition.

Definition 2 Let $(\mathcal{S}, p)$ be a probability space and $A, B$ two events in $\mathcal{S}$. The partition $\left\{C_{i}\right\}_{i \in I}$ of $\mathcal{S}$ is said to be a Reichenbachian common cause system (RCC system for short) for the pair $A, B$ if the following two conditions are satisfied

$$
\begin{gathered}
p\left(A \cap B \mid C_{i}\right)=p\left(A \mid C_{i}\right) p\left(B \mid C_{i}\right) \quad \text { for all } \quad i \in I \\
{\left[p\left(A \mid C_{i}\right)-p\left(A \mid C_{j}\right)\right]\left[p\left(B \mid C_{i}\right)-p\left(B \mid C_{j}\right)\right]>0 \quad(i \neq j)}
\end{gathered}
$$

The above definition is a natural generalization of Reichenbach's original definition of common cause to the case when more than one single factor contributes to a correlation. The cardinality of the index set $I$ (i.e. the number of events in the partition) is called the size of the RCCS. Since $C, C^{\perp}$ with a Reichenbachian common cause $C$ is a RCCS of size 2, we call a RCCS proper if its size is greater than 2 .

The next proposition shows that a Reichenbachian common cause system also has explanatory power exactly in the sense in which a single common cause does:

Proposition 2 Let the partition $\left\{C_{i}\right\}_{i \in I}$ of $\mathcal{S}$ be a Reichenbachian common cause system for the pair $A, B$. Then the elements $A$ and $B$ are positiely correlated.

Proof: The statement in the proposition is an immediate corollary of Lemma and the definition of the notion of Reichenbachian common cause system.

\section{Existence and uniqueness of Reichenbachian common cause systems}

It is not obvious that proper Reichenbachian common cause systems exist. It is not difficult however to give an example of a probability space $(\mathcal{S}, p)$ containing a pair of correlated events $A, B$ for which there exists no common cause in $\mathcal{S}$ but there exists in $\mathcal{S}$ a Reichenbachian common cause system of size 3 (see [8]). This example also shows that the intuition mentioned in Section 2 is correct: there are cases when an explanation of a correlation with the help of a single common cause is impossible within the bounds of a given event structure; yet the event structure is rich enough to contain a proper RCCS that can explain the correlation. It is not difficult however to find probability spaces that contain neither a (proper) common cause nor a proper Reichenbachian common cause system. As it was mentioned in Section 2 it is known that such common cause incomplete probability spaces can always be extended in such a way that the extension contains a common cause of the given correlation. More is true, however: on can show that any probability space can be extended in such a way that the larger probability space contains a Reichenbachian common cause system consisting of a large number of events. Before spelling out the precise proposition let us recall the definition of an extension $\left(\mathcal{S}^{\prime}, p^{\prime}\right)$ of a probability space $(\mathcal{S}, p)$ :

Definition $3\left(\mathcal{S}^{\prime}, p^{\prime}\right)$ is called an extension of $(\mathcal{S}, p)$ if there exists an injective lattice homomorphism (embedding) $h: \mathcal{S} \rightarrow \mathcal{S}^{\prime}$ (preserving also the orthocomplementation) such that

$$
p^{\prime}(h(X))=p(X) \quad \text { for all } \quad X \in \mathcal{S}
$$

Proposition 3 Let $(\mathcal{S}, p)$ be a classical probability space and $n$ be an arbitrary finite natural number greater than 2. There exists then an extension $\left(\mathcal{S}^{\prime}, p^{\prime}\right)$ of $(\mathcal{S}, p)$ such that $\left(\mathcal{S}^{\prime}, p^{\prime}\right)$ contains a Reichenbachian common cause system of size $n$. 
(See [8] for the long and tedious proof of this proposition.)

Proposition 3 shows that RCCS's of arbitrary finite size exist.

Problem Do Reichenbachian common cause systems of countably infinite size exist?

The answer to the above question is not known; we conjecture that RCCS's of infinite size also exist.

A given correlation $\operatorname{Corr}(A, B)$ in probability space $(\mathcal{S}, p)$ can possess two or more Reichenbachian common causes. Similarly, a correlation can have more than two proper Reichenbachian common cause systems (this being a consequence of Proposition 3); however, the different RCCS's cannot be arbitrarily located in the event structure $\mathcal{S}$. To formulate the proposition constraining the location of RCCS's in $\mathcal{S}$, consider the set $\mathbf{P}$ of all partitions of $\mathcal{S}$. Let $P_{1}=\left\{C_{i}^{1}\right\}_{i \in I}$ and $P_{2}=\left\{C_{j}^{2}\right\}_{j \in J}$ be two partitions in $\mathbf{P}$. The partition $P_{1}$ is defined to be finer than $P_{2}$ (equivalently: $P_{2}$ is defined to be coarser than $P_{1}$ ) (notation $\left.P_{1} \preceq P_{2}\right)$ if for every $C_{i}^{2} \in P_{2}$ there exist $C_{j_{l}^{i}}^{1} \in P_{1}\left(j_{l}^{i} \in L \subseteq J\right)$ such that $C_{i}^{2}=\cup_{j_{l}^{i} \in L} C_{j_{l}^{i}}^{1}$. $P_{1}$ is called strictly finer (coarser) than $P_{2}$ if $P_{1}$ is finer (coarser) than $P_{2}$ and $P_{1} \neq P_{2}$. The relation $\preceq$ is a partial ordering on $\mathbf{P}$, and the terminology "(strictly) finer" and "(strictly) coarser") applies to RCCS's as well since RCCS's are partitions.

Proposition 4 If $\left\{C_{i}\right\}_{i=1}^{i=n}$ is a Reichenbachian common cause system in $(\mathcal{S}, p)$ for the pair $A, B$, then there exists in $(\mathcal{S}, p)$ neither strictly finer nor strictly coarser Reichenbachian common cause system for $A, B$.

Proof: Assume that $\left\{C_{j}^{2}\right\}_{j=1}^{j=m}$ is a RCCS strictly coarser than the $\operatorname{RCCS}\left\{C_{i}^{1}\right\}_{i=1}^{i=n}(n>m)$. There exist then a $C_{j}^{2}$ such that for some $C_{i_{l}^{j}}^{1} \in P_{1}$ with $i_{l}^{j} \in L$ and $L$ having the cardinality of at least 2, we have $C_{j}^{2}=\cup_{i_{l}^{j} \in L} C_{i_{l}^{j}}^{1}$. Consider the probability measure space $\left(\mathcal{S}_{C_{j}^{2}}, p\left(\bullet \mid C_{j}^{2}\right)\right)$ where

$$
\mathcal{S}_{C_{j}^{2}}=\left\{X \cap C_{j}^{2} \quad \mid \quad X \in \mathcal{S}\right\}
$$

and where $p\left(\bullet \mid C_{j}^{2}\right)$ is the conditional probability measure of $p$ with respect to the conditioning event $C_{j}^{2}$. By the definition of $\left\{C_{j}^{2}\right\}_{j=1}^{j=m}$ as a Reichenbachian common cause system, the events $A$ and $B$ are statistically independent with respect to the probability measure $p\left(\bullet \mid C_{j}^{2}\right)$, i.e.

$$
p\left(A \cap B \mid C_{j}^{2}\right)=p\left(A \mid C_{j}^{2}\right) p\left(B \mid C_{j}^{2}\right)
$$

On the other hand, the events $C_{i_{l}^{j}}^{1}\left(i_{l}^{j} \in L\right)$ form Reichenbachian common cause system in $\left(\mathcal{S}_{C_{j}^{2}}, p\left(\bullet \mid C_{j}^{2}\right)\right)$ with respect to the events $\left(A \cap C_{j}^{2}\right)$ and $\left(B \cap C_{j}^{2}\right)$; hence, by Proposition 2 there is a positive correlation between $\left(A \cap C_{j}^{2}\right)$ and $\left(B \cap C_{j}^{2}\right)$ in the measure $p\left(\bullet \mid C_{j}^{2}\right)$ i.e. we have

$$
p\left(\left(A \cap C_{j}^{2}\right) \cap\left(B \cap C_{j}^{2}\right) \mid C_{j}^{2}\right)>p\left(A \cap C_{j}^{2} \mid C_{j}^{2}\right) p\left(B \cap C_{j}^{2} \mid C_{j}^{2}\right)
$$

which contradicts (13). So the assumption of existence of two, different RCCS's that are in the finer-coarser relation has led to contradiction, so the proposition is proved.

\section{Concluding remarks}

We can express the content of Proposition 4 in the following way: any totally ordered subset of partitions in $(\mathcal{S}, p)$ contains only one Reichenbachian common cause system for a given fixed pair of events $A, B \in \mathcal{S}$. So while there may exist many RCCS's for a given correlated pair, the different RCCS's provide different sorts of explanations of the correlation between $A$ and $B$. In particular, different Reichenbachian common cause systems cannot be "put together" to form a "finer" RCCS that would provide a more "detailed" explanation of the correlation. This also implies that the partition of $(\mathcal{S}, p)$ generated in the natural manner by different Reichenbachian common causes $C_{i} \in \mathcal{S}$ does not yield a RCCS; hence the non-uniqueness of Reichenbachian common causes cannot be explained by saying that the different common causes are just coarse-grained manifestations of a deeper, finer underlying Reichenbachian common cause system.

Given two correlations $\operatorname{Corr}\left(A_{i}, B_{i}\right)>0(i=1,2)$ in $(\mathcal{S}, p)$, the event $C \in \mathcal{S}$ is called a common common cause of the two correlations if it is a common cause of both $\operatorname{Corr}\left(A_{1}, B_{1}\right)>0$ and $\operatorname{Corr}\left(A_{2}, B_{2}\right)>0$. It is known that common causes are not in general common common causes, i.e. that there exist two correlations in a probability space that cannot have a common common cause (see [7] and [1] for results concerning necessary and sufficient conditions implying the existsence of common common causes). The notion of a Reichenbachian common common cause system also is a meaningful concept and it would be interesting to find necessary and sufficient conditions for a set of correlations to have a Reichenbachian common common cause system. 
Motivated by considerations somewhat different from the one in this paper, the problem of a common cause system is raised also in [9], where a common cause system (called "multiple common cause") is defined to be a finite partition possessing the screening off property (eq. (9)) only (plus some mathematically not explicit requirement concerning the spatiotemporal location of the events $C_{i}(i=1,2, \ldots n)$ and $A, B$, see Definition 3.3 in [9]). Thus the notion of RCCS defined in the present paper is different from the one proposed in [9]. We wish to point out in this regard that without some requirement in addition to (9) - such as (10) -, the notion of common cause system becomes trivial: for instance, the set of atoms in any finite Boolean algebra form a partition for which the screening off condition (9) holds; hence all probability measure spaces with a finite Boolean algebra possess a common common cause system, which seems counterintuitive. A Reichenbachian common cause system as defined in the present paper is a much stronger notion.

Reichenbach's notion of common cause can naturally be adapted to quantum probability spaces $(\mathcal{L}, p)$, where $\mathcal{L}$ is a non-distributive, orthomodular lattice and where $p$ is an additive (generalized) bounded measure on $\mathcal{L}$ (see [12], [10], [11], [4], [6]; for some other attempts see [2] and [3]). The notion of Reichenbachian common cause system also can easily be generalized to the non-commutative case along the ideas followed in this paper. Problems and questions concerning non-commutative Reichenbachian common cause systems paralelling the ones treated here also can be formulated, no results are known, however, on the non-commutative case.

Acknowledgement: Work supported by OTKA, contract numbers: T 035234, TS 04089, T 024841, T 043642 and T 025880.

\section{References}

[1] D. Danks and C. Glymour: Linearity properties of Bayes nets with binary variables, in J. Breese and D. Koller (eds.), Uncertainty in Artificial Intelligence: Proceedings of the 17th Conference (UAI-2001), Morgan Kaufmann, San Francisco, 2001, 98-109

[2] G. Hofer-Szabó: The formal existence and uniqueness of the Reichenbachian common cause on Hilbert lattices International Journal of Theoretical Physics 36 (1997) 1973-1980

[3] G. Hofer-Szabó: Reichenbach's common cause definition on Hilbert lattices International Journal of Theoretical Physics 37 (1998) 435-443

[4] G. Hofer-Szabó, M. Rédei and L.E. Szabó: On Reichenbach's common cause principle and Reichenbach's notion of common cause

The British Journal for the Philosophy of Science 50 (1999) 377-3999

[5] G. Hofer-Szabó, M. Rédei and L.E. Szabó: Common cause completability of classical and quantum probability spaces

International Journal of Theoretical Physics 39 (2000) 913-919

[6] G. Hofer-Szabó, M. Rédei and L.E. Szabó: Reichenbach's Common Cause Principle: Recent results and open problems

Reports on Philosophy Nr. 20, (2000) 85-107

[7] G. Hofer-Szabó, M. Rédei and Szabó, L.E. Common-causes are not common common-causes manuscript, submitted, preprint: http://philsci-archive.pitt.edu

[8] G. Hofer-Szabó and M. Rédei: Reichenbachian common cause systems of arbitrary finite size exist manuscript, submitted

[9] T. Placek: Is Nature Deetrministic?, Jagellonian University Press, Cracow, 2000

[10] M. Rédei: Reichenbach's Common Cause Principle and quantum field theory Foundations of Physics 27 (1997) 1309-1321

[11] M. Rédei: Quantum Logic in Algebraic Approach, Dordrecht: Kluwer Academic Publishers, 1998

[12] M. Rédei and S.J. Summers: Local primitive causality and the common cause principle in quantum field theory

Foundations of Physics 32 (2002) 335-355

[13] H. Reichenbach: The Direction of Time, Los Angeles: University of California Press, 1956 